\title{
Initial performance of the BICEP2 antenna-coupled superconducting bolometers at the South Pole
}

J. A. Brevik ${ }^{\dagger a}$, R. W. Aikin ${ }^{a}$, M. Amiri ${ }^{d}$, S. J. Benton ${ }^{e}$, J. J. Bock ${ }^{f}, a$, J. A. Bonetti ${ }^{f}$ B. Burger ${ }^{d}$, C. D. Dowell ${ }^{f}, a$, L. Duband ${ }^{g}$, J. P. Filippini ${ }^{a}$, S. R. Golwala ${ }^{a}$, M. Halpern ${ }^{d}$, M. Hasselfield ${ }^{d}$, G. Hilton ${ }^{h}$, V. V. Hristov ${ }^{a}$, K. $\operatorname{Irwin}^{h}$, J. P. Kaufman ${ }^{i}$, B. G. Keating ${ }^{i}$, J. M. Kovac ${ }^{j}$, C. L. Kuo ${ }^{b, c}$, A. E. Lange ${ }^{a}$, E. M. Leitch ${ }^{k}$, C. B. Netterfield ${ }^{e}$, H. T. Nguyen ${ }^{f, a}$, R. W. Ogburn IV ${ }^{b, c}$ A. Orlando ${ }^{a, f}$, C. Pryke ${ }^{l}$, C. Reintsema ${ }^{h}$, S. Richter ${ }^{j}$, J. E. Ruhl ${ }^{m}$, M. Runyan ${ }^{a}$, C. Sheehy ${ }^{k, l}$, Z. Staniszewski ${ }^{a, f}$, R. Sudiwala ${ }^{n}$, J. E. Tolan ${ }^{b, c}$, A. D. Turner ${ }^{f}$, P. Wilson ${ }^{f}$, and C. L. Wong ${ }^{j}$

${ }^{a}$ California Institute of Technology, 1200 E. California Blvd., Pasadena, CA 91125 USA

${ }^{b}$ Stanford University, Stanford, 382 Via Pueblo Mall, CA 94305, USA

${ }^{c}$ Kavli Institute for Particle Astrophysics and Cosmology (KIPAC), Sand Hill Road 2575, Menlo Park, CA 94025, USA

${ }^{d}$ Department of Physics \& Astronomy, University of British Columbia, 6224 Agricultural Road, Vancouver, BC V6T1Z1, Canada

${ }^{e}$ Department of Physics, University of Toronto, Toronto, ON M5S 1A7, Canada

fJet Propulsion Laboratory, 4800 Oak Grove Dr., Pasadena, CA 91109, USA

${ }^{g}$ Service des Basses Températures, DRFMC, CEA-Grenoble, 17 rue des Martyrs, 38054

Grenoble Cedex 9, France

${ }^{h}$ NIST Quantum Devices Group, 325 Broadway, Boulder, CO 80305, USA

${ }^{i}$ University of California, San Diego, La Jolla, CA 92093, USA

${ }^{j}$ Harvard-Smithsonian Center for Astrophysics, 60 Garden Street, Cambridge, MA 02138

${ }^{k}$ Kavli Institute for Cosmological Physics, University of Chicago, 5640 South Ellis Avenue, Chicago, IL 60637, USA

${ }^{l}$ University of Minnesota, Minneapolis, MN 55455, USA

${ }^{m}$ Physics Department, Case Western Reserve University, Cleveland, OH 44106, USA

${ }^{n}$ Dept. of Physics and Astronomy, University of Wales, Cardiff, CF24 3YB, Wales, UK

\begin{abstract}
We report on the preliminary detector performance of the BICEP2 mm-wave polarimeter, deployed in 2009 to the South Pole. BICEP2 is currently imaging the polarization of the cosmic microwave background at $150 \mathrm{GHz}$ using an array of 512 antenna-coupled superconducting bolometers. The antennas, band-defining filters and transition edge sensor (TES) bolometers are photolithographically fabricated on 4 silicon tiles. Each tile consists of an $8 \times 8$ grid of $\sim 7 \mathrm{~mm}$ spatial pixels, for a total of 256 detector pairs. A spatial pixel contains 2 sets of orthogonal antenna slots summed in-phase, with each set coupled to a TES by a filtered microstrip. The detectors are read out using time-domain multiplexed SQUIDs. The detector pair of each spatial pixel is differenced to measure polarization. We report on the performance of the BICEP2 detectors in the field, including the focal plane yield, detector and multiplexer optimization, detector noise and stability, and a preliminary estimate of the improvement in mapping speed compared to BICEP1.
\end{abstract}

Keywords: Cosmic microwave background, polarization, TES, noise, inflation, gravitational waves, cosmology

$\dagger$ Corresponding author: J. A. Brevik, 1200 E. California Blvd, MC: 367-17, Pasadena, CA 91125. E-mail: justusb@caltech.edu

Millimeter, Submillimeter, and Far-Infrared Detectors and Instrumentation for Astronomy V, edited by Wayne S. Holland, Jonas Zmuidzinas, Proc. of SPIE Vol. 7741, 77411H (c) 2010 SPIE · CCC code: $0277-786$ X/10/\$18 $\cdot$ doi: 10.1117/12.857861 


\section{INTRODUCTION}

The next frontier in cosmic microwave background (CMB) observation is the detection of the signature of inflation left in the polarized radiation remnant of the early universe. The theorized initial period of accelerated expansion of the universe is predicted to have given rise to a primordial gravitational wave background, inducing a faint but distinctive signal in the polarization of the CMB. ${ }^{1,2}$ The polarization pattern from this B-mode signal is uniquely divergence-free, and occurs at a much smaller level than the curl-free E-mode polarization first detected by the DASI experiment. ${ }^{3}$ The B-mode amplitude is parametrized by the ratio of the tensor to scalar perturbations to the metric tensor that source polarization, $r \sim \frac{\mathrm{T}}{\mathrm{S}}$. It has yet to be detected, but a current limit of $r<0.24$ has been set indirectly by WMAP7 from the contribution made to the temperature anisotropy at large angular scales. ${ }^{4}$ A direct constraint of $r<0.72$ has been made from B-mode polarization from the first two years of BICEP1 data. ${ }^{5}$

Sophisticated new instruments and techniques must be developed in order to pursue B-mode polarization. Greater sensitivity is necessary to integrate deeply enough to detect the $<0.1 \mu \mathrm{K}_{\mathrm{RMS}}$ signal. Instrumental systematics pose a challenge in their ability to create polarization artifacts that could obscure B-modes, and must be carefully controlled to meet the stringent requirements of B-mode detection. Monolithic detector arrays provide a great improvement of instrumental sensitivity through higher pixel counts. Photolithographic fabrication yields intrinsically well-controlled beams and polarization detection, and allows for extendable frequency coverage.

\section{INSTRUMENT}

BICEP2 is a ground-based CMB polarimeter utilizing a new generation of antenna-coupled transition edge sensor (TES) bolometers. It has been designed with the explicit goal of detecting B-mode polarization, with an emphasis on careful control of systematics. It is a monochromatic $(150 \mathrm{GHz})$, on-axis refractive telescope with a $25 \mathrm{~cm}$ aperture. The small aperture allows for careful control and characterization of instrumental systematics. The degree-size beams of BICEP2 allow it to integrate deeply at the angular scale at which the primordial B-mode power is expected to peak. The optics consist of a series of cooled, anti-reflection coated infrared blocking filters and lenses, optimized for a single frequency as described in Aikin et al. ${ }^{6}$

BICEP2 was deployed in December 2009 to the South Pole, Antarctica, and is currently funded for observation through the end of 2011. The high, dry and stable atmosphere and long polar night yield ideal conditions for long-integration, mm-wave observations. Additionally, the South Pole gives access to a unique patch of sky, the Southern Hole, where polarized foreground confusion from synchrotron radiation and especially thermal dust emission is much lower than the mean sky. For a single $150 \mathrm{GHz}$ receiver, detection of B-mode polarization should not be obscured in this field for $r>0.03$. The $150 \mathrm{GHz}$ spectral band has been designed to complement the band of reduced atmospheric emission at the South Pole. An overview of the instrument and the observational strategy are discussed in Ogburn et al. ${ }^{7}$

In late 2010, BICEP2 will be joined by the Keck array, a set of three monochromatic telescopes that will eventually add frequency coverage at 100 and $220 \mathrm{GHz} .{ }^{8}$ The multiple frequency bands are necessary for discrimination of the primordial B-mode from the frequency-dependent Galactic foregrounds.

The range of multipole moments investigated by BICEP2 is $\ell=30-300$, with primordial B-mode peaking at $\ell \sim 80$. With a scan rate of $2.8^{\circ} / \mathrm{s}$, this corresponds to a science band of $0.5-5 \mathrm{~Hz}$.

The receiver utilizes photolithographically fabricated, antenna-coupled superconducting bolometer arrays described in Kuo et al. ${ }^{9}$ The detector arrays are fabricated in the Micro Devices Laboratory at the Jet Propulsion Laboratory. The four $150 \mathrm{GHz}$ detector tiles in the focal plane have an $8 \times 8$ spatial pixel format, yielding a total of 256 detector pairs. A $\sim 7 \mathrm{~mm}$ spatial pixel includes two sets of independent phased-array planar slot antenna arrays, one per orthogonal polarization. Each antenna array is filtered and read out by a single transition edge sensor (TES), giving a total of 512 bolometers. Current signals from the voltage-biased TESs are read out using time-domain multiplexed superconducting quantum interference devices (SQUIDs). ${ }^{10,11}$ Automated SQUID tuning, multiplexing and data output is managed by the Multi-Channel Electronics (MCE) system, developed at the University of British Columbia. ${ }^{12}$ 

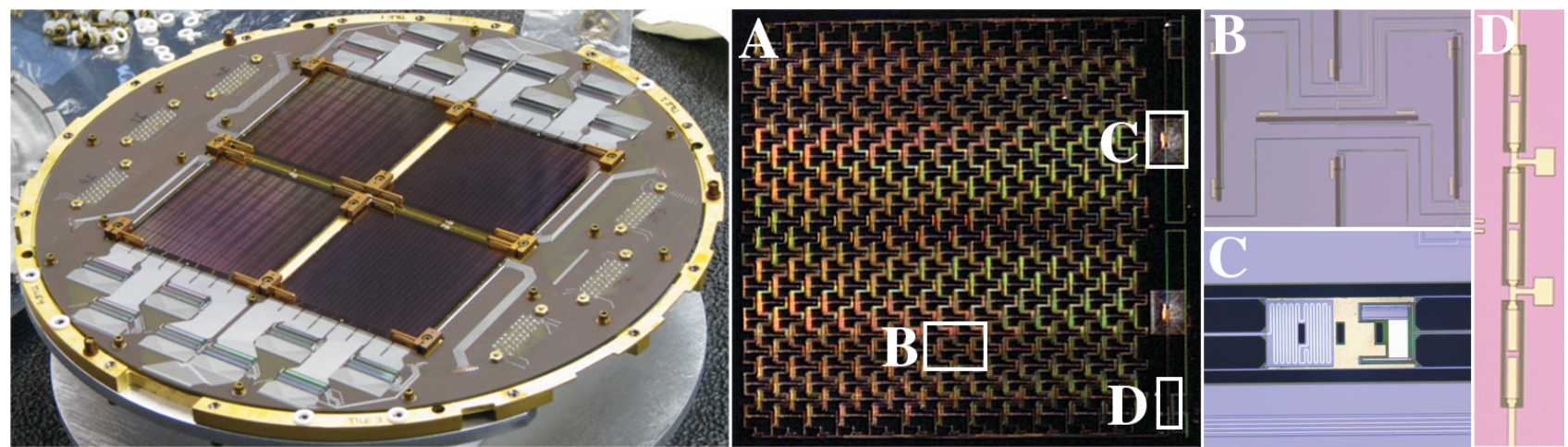

Figure 1. The BICEP2 focal plane with 4 detector tiles and multiplexed readout. One of the focal plane pixels (A), containing two orthogonal planar slot antenna arrays (B), with one TES bolometer per polarization (C), and a banddefining microstrip filter (D).

\subsection{FOCAL PLANE}

The BICEP2 focal plane includes a $27 \mathrm{~cm}$ diameter isothermal copper detector plate, four detector tiles each with a z-cut crystal quartz anti-reflection (AR) tile, 16 sets of NIST-fabricated SQUID multiplexer and Nyquist interface chips, a printed circuit board for routing of electrical connections, beryllium-copper spring clips to fix the arrays and AR tiles to the detector plate, and a superconducting niobium quarter-wave backshort. Many improvements to the focal plane and detectors took place prior to deployment of the receiver to the South Pole, as described in Orlando et al. ${ }^{13}$

The focal plane is cooled to $250 \mathrm{mK}$ by a Duband 3-stage sorption fridge, and is temperature controlled to $280 \mathrm{mK}$. This provides a stable bath temperature for the TESs and SQUID multiplexer chips, well below their superconducting transition temperatures. Fluctuations of the TES bath temperature resemble false changes in input power to the antennas, so the temperature of the detector plate is carefully controlled.

A focal plane pixel includes two orthogonal planar slot antenna arrays for beam formation, a filtered microstrip for spectral band definition, and a bolometer. Each bolometer consists of a detector island separated from the array by silicon nitride legs, a lossy gold meander for thermalizing power from the antenna on the island, a $\mathrm{Ti}$ and an Al TES in series, a layer of gold, and holes to promote the etching and release of the islands. The legs, along with the power input and TES output lines, determine the thermal conductance of the bolometer. The TES is composed of two thin films of $\mathrm{Ti}$ and $\mathrm{Al}$ in series, having target transition temperatures of $450 \mathrm{mK}$ and $1 \mathrm{~K}$ respectively. This allows the bolometers to function under the low $\sim 20 \mathrm{~K}$ optical loading conditions of the South Pole on the Ti transition, while also allowing for operation using the Al transition under high loading conditions, such as in the laboratory. A layer of gold is deposited on the island to increase the heat capacity of the bolometers, slowing them down to ensure stability of the detectors.

The slots of the antennas couple to the incident optical power, and electrical power from the slots is summed in-phase, transmitted along a microstrip line through a band-defining lumped-element filter and then to a TES island, where it is thermalized by a lossy gold meander. The current output of a voltage-biased TES is relayed to a Nyquist chip, by way of superconducting aluminum traces on the focal plane printed circuit board. The Nyquist chips include $1.35 \mu \mathrm{H}$ inductors for limiting the output bandwidth of the TESs, as well as $3 \mathrm{~m} \Omega$ shunt resistors for biasing them. Each TES is inductively coupled to a first-stage SQUID on a multiplexer chip. Flux feedback applied to the first-stage SQUID linearizes it and serves as the detector output for the channel.

Each of the 16 sets of Nyquist and multiplexer chips reads out 32 TES-coupled channels as well as a dark SQUID channel, giving a 33-row by 16-column multiplexing architecture. The 33 first-stage SQUIDs on one multiplexer chip are inductively coupled to a single second-stage SQUID on the chip, which is then relayed to a 100-element SQUID series array, located at $4 \mathrm{~K}$. The output of the second-stage SQUID is coupled to the input of the series array by a transformer that is available in 1-turn or 3-turn format. The choice is a balance between settling times of the multiplexer, affecting the multiplexed readout rate and ultimately the level of aliased noise; and the gain of the SQUID amplifier system, affecting the level of the amplifier noise referred to input. BICEP2 
uses 3-turn series arrays with little degradation of the settling times observed for the higher inductance. The TES shunt resistors on the Nyquist chips are biased in series by one pair of TES bias lines for each of the 16 sets of chips. The bias applied to maintain the TESs in their superconducting transition is thus a compromise for the 32 bolometers it serves.

During normal observation the MCE is configured for a multiplexed readout rate of $15.5 \mathrm{kHz}$. A 4-pole Butterworth filter with $f_{3 \mathrm{~dB}}=38.7 \mathrm{~Hz}$ is applied to the data by the MCE prior to downsampling to $100 \mathrm{~Hz}$. The maximum readout rate is limited by settling times in the SQUID multiplexer. A higher multiplexed readout rate would reduce the contribution of aliasing of out-of-band noise to the total noise level and may be pursued in the future if cross-talk is not introduced between multiplexer channels.

\subsection{DETECTORS}

An important parameter of a detector is its thermal conductance at the transition temperature $\left(\mathrm{G}_{\mathrm{c}}\right)$. This determines its saturation power, the amount of power that can be thermalized in the bolometer before electrothermal feedback can no longer maintain it in the superconducting transition. Given the optical loading expected from the receiver optics and from the CMB and atmosphere for our observation field, and given the desired margin or safety factor of additional loading, one arrives at a target value for $G_{c}$. Apart from the photon noise in the detector, the phonon noise has the largest impact on the unaliased noise levels within the science band. Thus, $\mathrm{G}_{\mathrm{C}}$ should be minimized to reduce detector noise but not be so low as to risk saturation of the detectors. BICEP2 was designed for roughly equal optical and electrical power, or a safety factor of 2 . The total loading is expected to be $\sim 4.5 \mathrm{pW}$, so the designed saturation power is $9.0 \mathrm{pW}$. For a target $\mathrm{T}_{\mathrm{C}}$ of $450 \mathrm{mK}$ and anticipated base temperature of $250 \mathrm{mK}$, the target $\mathrm{G}_{\mathrm{c}}$ is thus $80 \mathrm{pW} / \mathrm{K}$. The measured thermal conductances range from $80-$ $130 \mathrm{pW} / \mathrm{K}$, with the mean conductance of two tiles meeting the $80 \mathrm{pW} /$ Ktarget, while the other two are higher and have more variation.

The superconducting transition temperature $\left(\mathrm{T}_{\mathrm{c}}\right)$ of the Ti TESs was targeted at $450 \mathrm{mK}$. The measured transition temperatures range from $500-530 \mathrm{mK}$.

The speed of the detectors has an impact on their stability. It is necessary that the electrical and thermal time constants of the detectors be well separated to avoid their interaction, which gives rise to oscillations in the detectors. As the detectors are biased lower into the transition, their electrical time constants increase while their thermal time constants decrease, causing the poles to converge and the detectors to oscillate. The speed of the detectors has been measured by introducing a square-wave signal to the detector bias lines, with an electrical response to the change in applied bias and a thermal response to the heat introduced on the island. The effective thermal time constants have been estimated from fits to the stepped response. With $2.5 \mu \mathrm{m}$ of gold added to the detector islands to increase the heat capacity, the time constants are $>1$ ms even low in the transition. The high heat capacity and low thermal conductance of the bolometers have given a sufficiently high thermal time constant that the detectors do not oscillate for our choice of operating biases. Furthermore, although they have been slowed down by the gold, the detectors are still sufficiently fast for our scan speed and their transfer functions are flat within the science band, showing no low-frequency roll-off.

\section{DETECTOR OPTIMIZATION \& YIELD}

Detector biases have been selected in order to minimize the instrumental noise-equivalent temperature (NET), while ensuring stability of the detector responsivities. To determine the bias that minimizes a column's NET, we have combined measurements of current noise for each detector with an estimate of its optical responsivity. The responsivity is derived from the calibration elevation nods described in Ogburn et al. to scale the current to temperature units. ${ }^{7}$ The procedure, described below, results in profiles of per-column sensitivities versus applied bias as shown in Figure 2. The spread in the minima for the NET profiles is due to the difference in thermal conductances for the 4 detector tiles. Tiles 3 and 4 correspond to columns 8 through 15 , whose minima are tightly grouped and require lower applied bias. Detectors on these tiles have lower and more consistent thermal conductances, so less electrical power is needed to maintain them in the transition and there is less spread in the optimal biases. In practice, the biases being used for observations are lower than those given by the minima in Figure 2 to ensure the detectors are well into their transitions and have stable optical responsivities, and are not close to the regions of high NET slope near the minima. However, by using lower biases the instrumental 


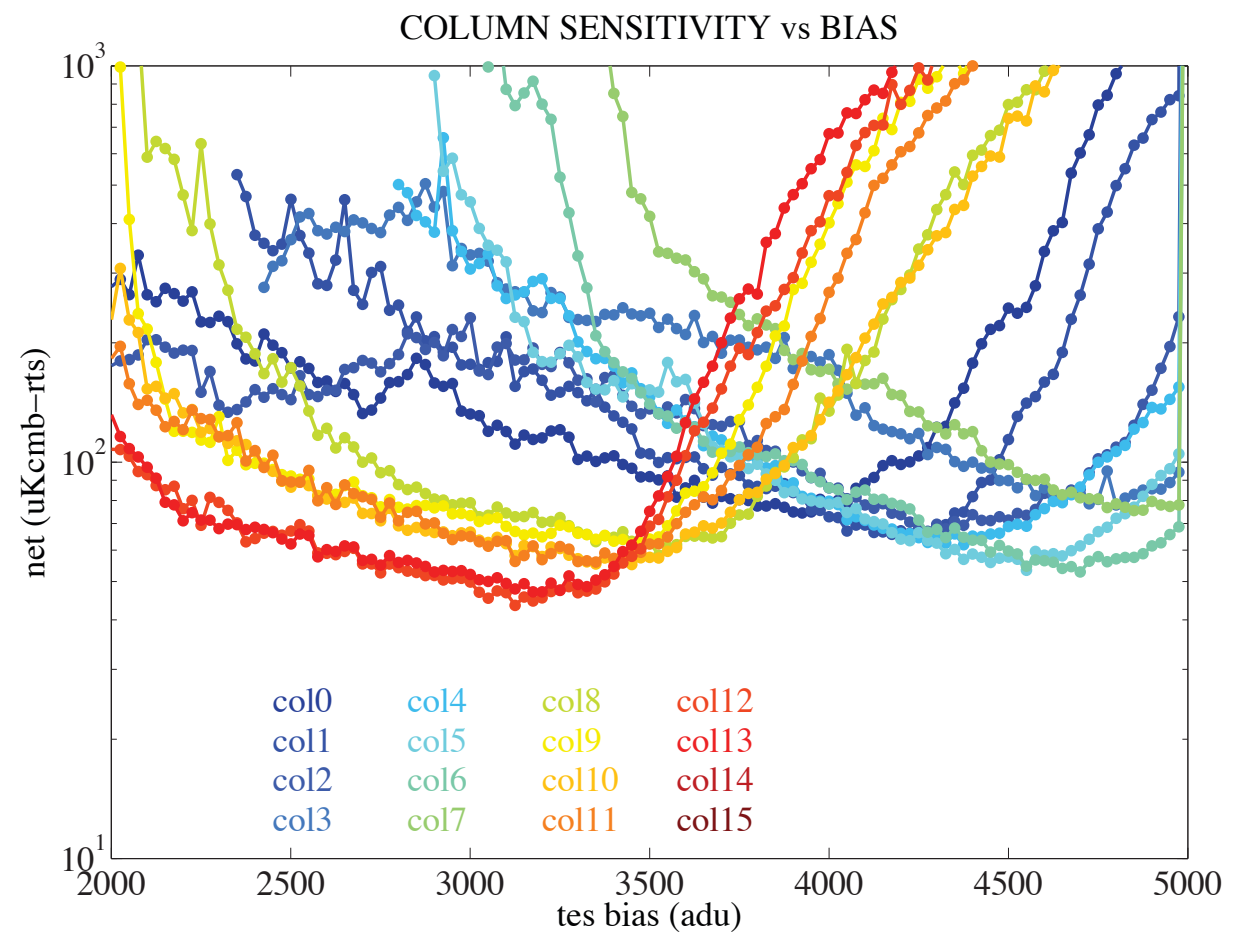

Figure 2. An estimate of NETs for the 16 detector columns versus applied TES bias in ADU, for determining the optimal detector biases. The total NET for a column is determined by inverse variance weighting the NETs for the 32 detectors biased in that column. The actual biases used for the different columns are lower than the minima in order to ensure stability of the optical responsivities and move away from the regions of highly sloped NET near the minima.

NET is higher than optimal by around $11 \%$. The biases may be increased in the future to their optimal values to regain the sensitivity, after further studies of responsivity stability have been made.

To estimate NETs from noise spectra and elevation nods, the TESs are driven normal and the detector bias is stepped down finely through the transition. With the telescope pointed at an elevation of $55^{\circ}$, representative of the loading during normal observations, 60 -second time streams are acquired for all detector channels at each bias step. From these, power spectral density estimates are made to determine the noise level in the science band. In addition, a standard elevation nod of $1.2^{\circ}$ is executed symmetrically about $55^{\circ}$ to introduce a $\sim 220$ $\mathrm{mK}_{\mathrm{CMB}}$ modulation of optical load on the detectors, assuming a zenith temperature of $12.3 \mathrm{~K}_{\mathrm{CMB}}$. The fit of the detector response to the change in airmass yields a scaling of current to CMB temperature units. The percolumn sensitivity at each bias step is then calculated by inverse variance weighting the individual NETs of the functioning detectors within a column. This method is currently only used to optimize the detectors and not to determine their per-detector sensitivities, due to uncertainty in the choice of zenith temperature.

Analysis of load curves executed during observation show that for the standard biases the mean operational resistance of the detectors is $61 \mathrm{~m} \Omega$ or $82 \%$ in fractional resistance $\left(\mathrm{R} / \mathrm{R}_{\mathrm{N}}\right)$. In addition, the mean electrical power is $\sim 6 \mathrm{pW}$ for tiles 3 and 4 and $\sim 13 \mathrm{pW}$ for tiles 1 and 2 . Measurements of optical loading under standard observation conditions and at the mean elevation of the BICEP2 field have yielded $\mathrm{Q}_{\mathrm{LOAD}} \approx 4-6 \mathrm{pW}$. Tiles 3 and 4 have lower conductance and saturation power, and seem to be operating with roughly equal optical and electrical power. The higher conductance tiles 1 and 2 with the higher saturation power, have a much larger margin for optical loading and are operating with a 1:2 ratio of optical to electrical power.

The SQUID tuning parameters have been optimized in order to maximize the number of operational channels, at the expense of some of the end-to-end amplifier gain. The amplifier noise referred to input is thus larger, but as will be discussed below, it is subdominant to other noise sources.

Of the 512 TESs on the BICEP2 focal plane, 441 are optically-coupled and operational (86\%). It is possible that more will be flagged for poor performance in the future. Based on electrical testing of the arrays prior to integration in the focal plane, 507 detectors were operational for a fabrication yield of $99 \%$. The microstrip coupling the antenna to the TES has been disconnected for 12 detectors to create dark detectors to assess common-mode magnetic and thermal pickup of the TESs. The SQUID multiplexer chips are cryogenically 


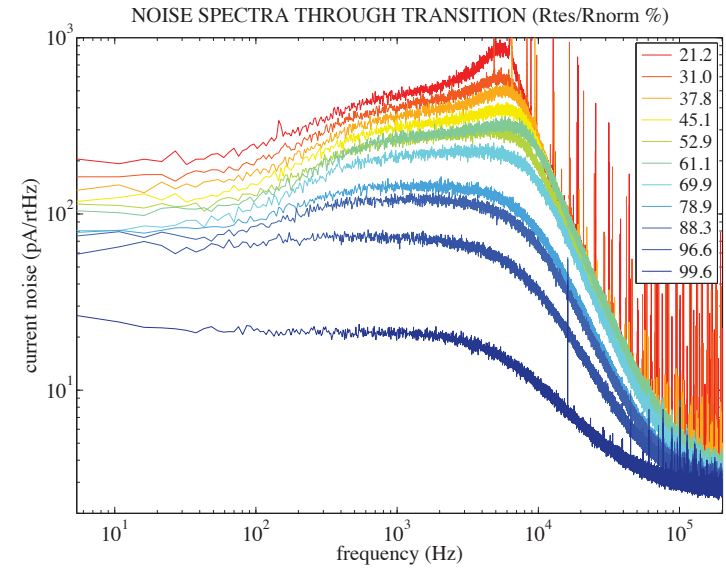

(a) Noise spectra through the transition

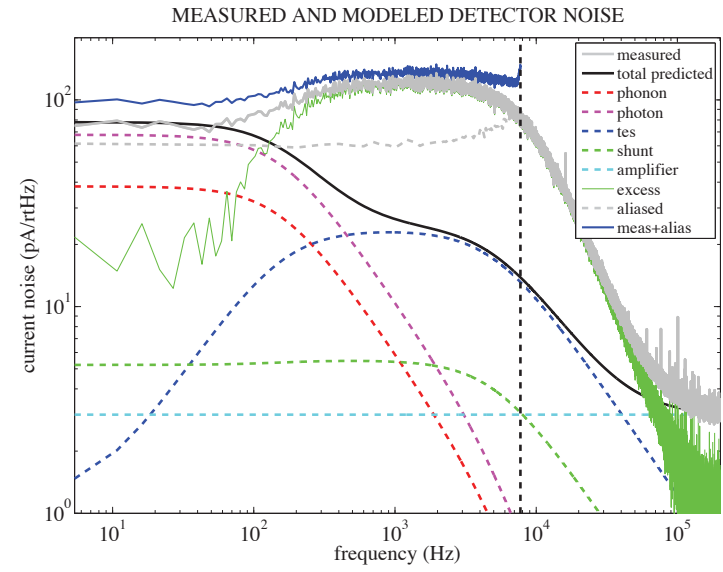

(b) Measured and modeled current noise

Figure 3. (a) Noise spectra through the transition for a characteristic detector, sampled at $400 \mathrm{kHz}$. As the detector is biased lower in the transition an excess noise plateau develops and eventually the detector begins to oscillate. For the standard observation bias, this detector will operate at $86 \%$ fractional resistance. (b) Measured and modeled current noise for the characteristic detector at the observation bias. The aliased noise level is calculated from the measured noise spectrum above the Nyquist frequency, shown as the dashed vertical line. The total predicted noise is calculated from the noise model using the measured detector parameters.

screened prior to integration, which revealed a loss of 6 channels. After the multiplexers were integrated and the SQUIDs were properly biased and tuned, various issues with the first-stage SQUIDs have further reduced the number of operational channels by 32 . In addition, an electrical connection issue on one multiplexer column has eliminated capability of reading out its 32 optically-coupled channels. Finally, a few detectors are unusually noisy and have been flagged and removed from analysis. Many of these issues overlap, but the net result is that 71 antenna-coupled TESs in 60 detector pairs are nonoperational. Due to pair differencing 196 detector pairs will be used in analysis, for a total of 392 operational detectors. These yield issues result in a 14\% reduction in the sensitivity of the receiver compared to the ideal.

\section{DETECTOR NOISE COMPONENTS}

In this section we summarize the contributions to the total noise of the BICEP2 devices. The noise components include photon, phonon (thermal fluctuation), Johnson, excess and amplifier noise. In order to determine the components of the total noise, the detectors must be sampled above the standard $15.5 \mathrm{kHz}$ rate in order to assess the high-frequency contributions and prevent obscuration from aliasing. Special data taking modes of the MCE were used to read out the detectors at $400 \mathrm{kHz}$ and $50 \mathrm{MHz}$, with downsampling and filtering turned off. Noise spectra were produced from the time streams, and aliasing calculations were made to simulate the impact of the noise above $7.75 \mathrm{kHz}$ in the science band.

In Figure 3(a) the $400 \mathrm{kHz}$-sampled noise spectra of a characteristic optically-coupled detector are shown through the transition. Moving down through the transition, the low-frequency noise levels increase and an excess noise plateau develops. Very low in the transition the detector begins to oscillate, with a resonance in the spectra forming around $6 \mathrm{kHz}$. In Figure 3(b) the noise spectrum of the same detector at the standard observation bias is shown. The various noise contributions predicted from the detector parameters have been overlayed with the measured spectrum, along with the aliased noise level calculated from the measured spectrum.

The characteristic optically-coupled detector shown in Figure 3(b) is operating at a fractional resistance of $86 \%$. Its noise has been modeled using detector parameters $\left(T_{c}, G_{c}, \beta\right)$ derived from load curves taken at a range of base temperatures, detector operating bias parameters $\left(\mathrm{R}_{\text {TES }}, \mathrm{I}_{\text {TЕS }}\right)$ derived from routine load curves during observations, heat capacity estimates from thermal time constant measurements, an estimate of $\alpha$ from resistance versus temperature measurements, along with additional inputs described below. The measured noise 
agrees with the total predicted noise at low frequency when modeled with $6 \mathrm{pW}$ of loading. This value is consistent with the $4-6 \mathrm{pW}$ of loading measured during the austral summer.

The same noise model has been applied to noise spectra of dark detectors for which the photon noise is negligible, and the low-frequency agreement of the measured and modeled noise is also quite good. This suggests that the detector bias parameters have been accurately measured, which would impact the responsivity and scaling of the NEC. For comparison with general estimates of noise below, the significant low-frequency modeled noise components are $\mathrm{NEC}_{\text {тF⿻ }} \approx 38 \mathrm{pA} / \sqrt{\mathrm{Hz}}, \mathrm{NEC}_{\text {рнотоN }} \approx 68 \mathrm{pA} / \sqrt{\mathrm{Hz}}, \mathrm{NEC}_{\text {ALASED }} \approx 60 \mathrm{pA} / \sqrt{\mathrm{Hz}}$, for a $\mathrm{NEC}_{\text {тота丿 }} \approx$ $98 \mathrm{pA} / \sqrt{\mathrm{Hz}}$ including aliased noise. The measured noise in Figure 3(b) that extends above the Nyquist frequency, shown with the vertical dashed line, has been aliased back in-band and added to the measured noise level. The resulting noise trace represents the expected noise levels for the multiplexed data, and agrees with data that has been taken in the standard readout mode.

In the remainder of this section the expected levels of each noise contribution in the science band are estimated from the median measured detector parameters of the BICEP2 devices for the standard observation conditions. The estimates can be compared to the noise modeled for the characteristic detector. Where appropriate, a DC power-to-current responsivity of $\mathrm{s}_{\mathrm{r}}(0) \approx-1.38 \cdot 10^{6} \mathrm{~A} / \mathrm{W}$ has been used to convert between noise-equivalent power (NEP) and noise-equivalent current (NEC) referred to the input coil of the first-stage SQUID. This value has been derived from the median detector bias parameters $\left(\mathrm{R}_{\mathrm{TES}}, \mathrm{I}_{\mathrm{TES}}\right)$ during observation, for the case of strong electro-thermal feedback $\mathrm{S}_{\mathrm{I}}(0)=\frac{-1}{\mathrm{I}_{\mathrm{TES}}\left(\mathrm{R}_{\mathrm{TES}}-\mathrm{R}_{\mathrm{SHUNT}}\right)} \cdot{ }^{14}$

The thermal fluctuation or phonon noise is attributed to thermodynamic fluctuations of the detector's thermal impedance. After photon noise, this is expected to make the largest contribution to the unaliased noise level in the science band for the target conductance. Using the median values of $\mathrm{G}_{\mathrm{c}} \approx 100 \mathrm{pW} / \mathrm{K}$ and $\mathrm{T}_{\mathrm{c}} \approx 520 \mathrm{mK}$ for the detector arrays an estimate of the phonon noise can be made from:

$$
\mathrm{NEP}_{\text {теN }}^{2}=4 \mathrm{k}_{\mathrm{B}} \mathrm{T}_{\mathrm{C}}^{2} \mathrm{G}_{\mathrm{C}} \mathrm{F}\left(\mathrm{T}_{\mathrm{c}}, \mathrm{T}_{\text {вАтн }}\right)
$$

where $\mathrm{F}\left(\mathrm{T}_{\mathrm{C}}, \mathrm{T}_{\text {ватн }}\right)$ is a factor that accounts for nonlinear thermal conductance and is estimated at $0.5 .^{14}$ This yields $\mathrm{NEP}_{\mathrm{TFN}} \approx 27 \mathrm{aW} / \sqrt{\mathrm{Hz}}$ and $\mathrm{NEC}_{\mathrm{TPN}} \approx 38 \mathrm{pA} / \sqrt{\mathrm{Hz}}$.

Johnson noise from the detectors and shunts contribute little to the unaliased noise within the science band. The detector Johnson noise could make a significant contribution, but is suppressed at low frequency by the loop gain in electro-thermal feedback. The DC contribution from these sources can be estimated from:

$$
\mathrm{NEP}_{\text {JOHNSON }}^{2}=\mathrm{NEP}_{\text {TES }}^{2}+\mathrm{NEP}_{\text {SHUNT }}^{2}=4 \mathrm{k}_{\mathrm{B}} \mathrm{T}_{\mathrm{C}} \mathrm{R}_{\mathrm{TES}} \mathrm{I}_{\mathrm{TES}}^{2} \frac{1}{\mathscr{L}_{\mathrm{I}}^{2}}+4 \mathrm{k}_{\mathrm{B}} \mathrm{T}_{\text {sHUNT }} \mathrm{R}_{\mathrm{SHUNT}} \mathrm{I}_{\mathrm{TES}}^{2} \frac{\left(\mathscr{L}_{1}-1\right)^{2}}{\mathscr{L}_{\mathrm{I}}^{2}}
$$

where $\mathscr{L}_{\mathrm{I}} \equiv \frac{\mathrm{P}_{\mathrm{TES}} \alpha}{\mathrm{G}_{\mathrm{C}} \mathrm{T}_{\mathrm{C}}}$ is the loop gain, and $\alpha$ is the sensitivity of the transition. ${ }^{14}$ Using the mean operational parameters of the detectors; $\mathrm{R}_{\text {теs }} \approx 61 \mathrm{~m} \Omega, \mathrm{I}_{\text {тве }} \approx 13.5 \mu \mathrm{A}, \mathscr{L}_{1} \approx 21.6$, along with $\mathrm{R}_{\text {sнимт }}=3 \mathrm{~m} \Omega$ and $\mathrm{T}_{\text {янитт }}=500 \mathrm{mK}$, the combined Johnson noise of the TES and shunt is estimated at $\mathrm{NEP}_{\text {Jонмsом }} \approx 4 \mathrm{aW} / \sqrt{\mathrm{Hz}}$ and $\mathrm{NEC}_{\text {Jонмsoм }} \approx 5$ $\mathrm{pA} / \sqrt{\mathrm{Hz}}$.

Excess noise has been observed in the BICEP2 detectors and has a large impact on the total aliased noise levels. A significant amount of power from the excess noise extends beyond the Nyquist frequency of our multiplexed readout. The single-pole Nyquist filters are expected to roll off the noise around $5-6 \mathrm{kHz}$ under standard operating biases. However, the filters do not roll off the excess noise very aggressively, and so a large amount is aliased back in-band. As the detector is biased lower into the transition the excess noise plateau rises. The noise power above the Nyquist frequency increases, increasing the level of aliased noise.

The excess noise is not included in the TES noise model, but can be estimated in our characteristic detector after the total predicted noise has been removed from the measured spectrum. As shown in Figure 3(b), the unaliased NEC of the excess noise at low frequency is negligible, but the aliased contribution is significant at $\sim 60 \mathrm{pA} / \sqrt{\mathrm{Hz}}$. From the figure it is clear that the high-frequency noise in the detector is dominated by excess noise, and thus so is the aliased noise. Performing aliasing calculations on the $400 \mathrm{kHz}$ spectra, the median total aliased noise levels for the detectors are $\mathrm{NEC}_{\text {EXcess } / \text { ALLAsing }} \approx 64 \mathrm{pA} / \sqrt{\mathrm{Hz}}$ and $\mathrm{NEP}_{\text {EXCEs/ } / \text { ALIAsing }} \approx 46 \mathrm{aW} / \sqrt{\mathrm{Hz}}$, for the standard readout rate. The median level agrees with the aliased noise in the characteristic detector. 

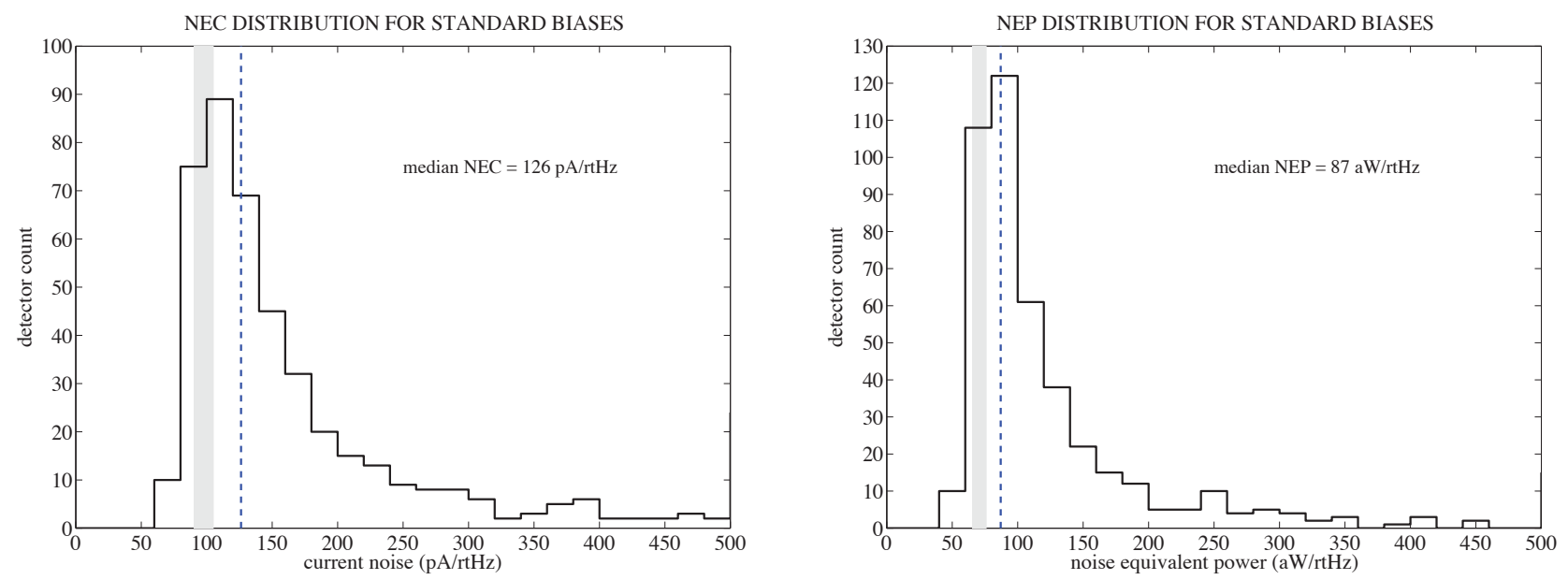

Figure 4. Histograms of the measured NECs and NEPs for the operational detectors at the standard observation biases, based on 60-second time streams acquired during the austral summer. The medians of the distributions are indicated by the dashed lines. The shaded regions show the range of NECs and NEPs expected from the noise model based on median measured detector parameters.

The amplifier noise contributed by the cold and warm electronics has been estimated by sampling each channel at $50 \mathrm{MHz}$, with flux-feedback turned off and the detectors superconducting. The noise spectra of the TES-coupled channels are rolled off by the Nyquist filters at $\sim 300 \mathrm{~Hz}$, down to the unaliased noise level of the the amplifier around $200 \mathrm{MHz}$. The spectra are then rolled off around $1 \mathrm{MHz}$ by anti-aliasing filters prior to digitization by the MCE's analog-to-digital converters. Using the closed-loop gain of the SQUID amplifiers, the noise can be referred to input to give the NEC of the amplifier. The mean of all the channels is $\mathrm{NEC}_{\text {MMP }} \approx 2$ $\mathrm{pA} / \sqrt{\mathrm{Hz}}$, in agreement with the design budget for amplifier noise. Performing the aliasing calculation on the 50 $\mathrm{MHz}$ spectra gives $\sim 25 \mathrm{pA} / \sqrt{\mathrm{Hz}}$ or $\sim 18 \mathrm{aW} / \sqrt{\mathrm{Hz}}$ for the mean aliased amplifier noise. This is already included in the aliased level above that is attributed mostly to excess noise and should not be included in the final detector noise total.

Photon noise, from shot noise and Bose noise, should be the largest contribution to the unaliased noise levels in the science band. The contribution can be estimated from:

$$
\mathrm{NEP}_{\text {PHOTON }}^{2}=\mathrm{NEP}_{\text {SHOT }}^{2}+\mathrm{NEP}_{\text {BOSE }}^{2}=2 \mathrm{~h} \nu \mathrm{Q}_{\mathrm{LOAD}}+\frac{2 \mathrm{Q}_{\mathrm{LOAD}}^{2}}{\nu \frac{\Delta \nu}{\nu}}
$$

where $\nu$ is the band center, $\frac{\Delta \nu}{\nu}$ is the fractional bandwidth, and $\mathrm{Q}_{\mathrm{LOAD}}$ is the optical load on the detectors. ${ }^{14}$ Preliminary measurements of the detector spectral response yielded a mean band center of $148 \mathrm{GHz}$, and fractional bandwidth of $25 \%$. For the range of optical loading $\mathrm{Q}_{\mathrm{LOAD}} \approx 4-6 \mathrm{pW}$ given in Section 3, the expected photon noise is $\mathrm{NEP}_{\text {рноток }} \approx 41-56 \mathrm{aW} / \sqrt{\mathrm{Hz}}$ or $\mathrm{NEC}_{\text {рноток }} \approx 56-77 \mathrm{pA} / \sqrt{\mathrm{Hz}}$. For this level of optical loading, the BICEP2 detectors are approaching background-limited performance if aliased noise is excluded. However, for some detectors the photon noise could be dominated by the $\sim 64 \mathrm{pA} / \sqrt{\mathrm{Hz}}$ contributed by the aliasing of excess noise.

Considering the contributions of each of these to the total noise, along with the aliasing:

$$
\mathrm{NEP}_{\text {TOTAL }}^{2}=\mathrm{NEP}_{\text {PHotoN }}^{2}+\mathrm{NEP}_{\text {TFN }}^{2}+\mathrm{NEP}_{\text {JоHNSON }}^{2}+\mathrm{NEP}_{\text {AMP }}^{2}+\mathrm{NEP}_{\text {EXEESS/ALLASIIG }}^{2}
$$

one would expect a $\mathrm{NEP}_{\text {тота }} \approx 68-78 \mathrm{aW} / \sqrt{\mathrm{Hz}}$ or $\mathrm{NEC}_{\text {тота }} \approx 93-107 \mathrm{pA} / \sqrt{\mathrm{Hz}}$. The characteristic detector of Figure 3(b) has a total NEC with aliasing of $\sim 98 \mathrm{pA} / \sqrt{\mathrm{Hz}}$, which agrees with the expectation from the noise model. In Figure 4 histograms of the measured NECs and NEPs for the operational detectors at the standard biases is shown. Noise spectra are derived from 60-second time streams acquired at the mean elevation of the observation field during the austral summer. The noise level within the science band is averaged to give the 
NEC. The NEPs are scaled from the measured NECs using the individual power-to-current responsivity of each detector, calculated from its bias parameters $\mathrm{R}_{\mathrm{TES}}$ and $\mathrm{I}_{\mathrm{TES}}$. The median measured NEC is $126 \mathrm{pA} / \sqrt{\mathrm{Hz}}$, while the median measured NEP is $87 \mathrm{aW} / \sqrt{\mathrm{Hz}}$. The ranges of NEP and NEC calculated from the median detector parameters, shown as the shaded regions in Figure 4, are lower than the median of the actual measured values shown as the vertical dashed lines. The noise data were acquired during the austral summer, so the measured values could be larger due to higher loading conditions and higher atmospheric noise levels.

The target NEP for the BICEP2 detectors is $\sim 77 \mathrm{aW} / \sqrt{\mathrm{Hz}}$, to achieve the target $330 \mu \mathrm{K}_{\text {смв }} \sqrt{\mathrm{s}}$ per-detector sensitivity. The median of the measured NEPs is $\sim 13 \%$ higher than the target. However, the range of NEP calculated from the median measured detector parameters suggests that the target may have been met, and that the measured NEPs reflect the noisier conditions of the austral summer.

\section{ALIASED NOISE REDUCTION}

Aliased excess noise appears to be dominating the noise of a significant fraction of the BICEP2 detectors. Based on estimates of noise from the median detector parameters, we expect a total NEP without aliasing in the range of $49-62 \mathrm{aW} / \sqrt{\mathrm{Hz}}$ for $4-6 \mathrm{pW}$ of loading. The expected range increases to $68-78 \mathrm{aW} / \sqrt{\mathrm{Hz}}$ when we include the median aliased contribution of $46 \mathrm{aW} / \sqrt{\mathrm{Hz}}$. The aliased noise could be reduced by a combination of increased Nyquist inductance and increased multiplexed readout rate.

Future science-grade focal planes could be produced for BICEP2 or the Keck array using larger Nyquist inductors. Inductances of $1.35 \mu \mathrm{H}$ were the largest available at the time of integration of the BICEP2 focal plane, and are currently limited to $2 \mu \mathrm{H}$ due to fabrication constraints. Increasing the Nyquist inductance increases the electrical time constants of the detectors, and requires a corresponding increase in the thermal time constants to prevent oscillations. With the lower limit of the thermal conductance fixed by optical loading, the heat capacity of the detectors must be increased. It should be possible to increase the heat capacity of the bolometers enough to accommodate $2 \mu \mathrm{H}$ inductors to maintain an adequate range of detector stability.

Increasing the multiplexed readout rate would certainly decrease the aliased noise level. The current readout rate was chosen conservatively to ensure there was no cross talk introduced by settling times in the SQUID multiplexer. Faster rates may be possible without introducing cross talk between channels. If not, it's possible that a hardware or software solution to reduce the settling times will be found. Based on aliasing calculations of the $400 \mathrm{kHz}$ noise spectra, increasing the readout rate to $20 \mathrm{kHz}$ will reduce the median aliased noise level to $36 \mathrm{aW} / \sqrt{\mathrm{Hz}}$, and increasing it to $30 \mathrm{kHz}$ will reduce it to $22 \mathrm{aW} / \sqrt{\mathrm{Hz}}$. Above a $20 \mathrm{kHz}$ readout rate the aliased noise will fall below the $41 \mathrm{aW} / \sqrt{\mathrm{Hz}}$ expected from photon noise for $4 \mathrm{pW}$ of loading.

\section{MAPPING SPEED}

We have made a preliminary estimate of the improvement in mapping speed of BICEP2 over BICEP1 based on maps made from 4 days $\left(\sim 1.7 \cdot 10^{5} \mathrm{~s}\right)$ of high quality data for each instrument. Taking the ratio of the variance in the maps gives the ratio of the mapping speeds. A preliminary estimate from this analysis suggests a factor of $8-10$ increase in mapping speed relative to BICEP1. This estimate includes the improvements in observation efficiency discussed in Ogburn et al., so it does not purely reflect the gain in instantaneous instrumental sensitivity. ${ }^{7}$

\section{CONCLUSION}

The BICEP2 instrument has been observing from the South Pole since February 2010 in pursuit of B-mode polarization. The instrument has been configured for optimal instrumental sensitivity, with the possibility of improvement in the future. The aliasing of excess noise may be reduced if a higher multiplexed readout rate is achieved. There are 32 channels lost to a wiring issue that will be fixed next summer, and more channels may be salvaged by further optimization of poorly performing first-stage SQUIDs. Finally, some of the performance lost by reducing the detector biases to ensure stability of the optical responsivity of the detectors may be recovered. Based on a preliminary analysis of mapping data, BICEP2 appears to improve the mapping speed of its predecessor BICEP1 by a factor of $8-10$. The gain is attributable to a combination of likely improvement to per-detector sensitivities, a fourfold increase in the number of functioning detectors, and increased observing efficiency. 


\section{ACKNOWLEDGMENTS}

BICEP2 has been made possible by support from the National Science Foundation, Grant No. ANT-0742818. Detector development has been made possible by the generous support of the Gordon and Betty Moore Foundation. We are grateful to have Steffen Richter as our 2010 South Pole winterover. The BiceP2 team would also like to thank the South Pole Station staff for logistical support. We thank our BICEP1, Keck Array, and

Spider colleagues for useful discussions and shared expertise. Finally we wish to thank Barbara Wertz and Kathy Deniston for their tireless logistical and administrative support.

\section{REFERENCES}

[1] Seljak, U. et al., "Signature of Gravity Waves in the Polarization of the Microwave Background," Phys. Rev. Lett. 78 (Mar 1997).

[2] Kamionkowski, M. et al., "A Probe of Primordial Gravity Waves and Vorticity," Phys. Rev. Lett. 78 (Mar 1997).

[3] Kovac, J. M. et al., "Detection of polarization in the cosmic microwave background using DASI," Nature $\mathbf{4 2 0}$, $722-787$ (2002).

[4] Komatsu, E. et al., "Seven-Year Wilkinson Microwave Anisotropy Probe (WMAP) Observations: Cosmological Interpretation," ArXiv (Feb 2010).

[5] Chiang, H. C. et al., "Measurement of cosmic microwave background polarization power spectra from two years of BICEP data," Astrophys. J 711, 1123-1140 (2010).

[6] Aikin, R. W. et al., "Optical performance of the BICEP2 telescope at the South Pole," These proceedings (2010).

[7] Ogburn, R. W. et al., "The BICEP2 CMB polarization experiment," These proceedings (2010).

[8] Sheehy, C. et al., "The Keck Array: a pulse tube cooled CMB polarimeter," These proceedings (2010).

[9] Kuo, C. L. et al., "Antenna-coupled TES Bolometer Arrays for CMB Polarimetry," SPIE Proceedings $\mathbf{7 0 2 0}$ (July 2008).

[10] de Korte, P. A. J., Beyer, J., Deiker, S., Hilton, G. C., Irwin, K. D., Macintosh, M., Nam, S. W., Reintsema, C. D., Vale, L. R., and Huber, M. E., "Time-division superconducting quantum interference device multiplexer for transition-edge sensors," Review of Scientific Instruments 74, 3807-3815 (Aug. 2003).

[11] Irwin, K. D., Vale, L. R., Bergren, N. E., Deiker, S., Grossman, E. N., Hilton, G. C., Nam, S. W., Reintsema, C. D., Rudman, D. A., and Huber, M. E., "Time-division SQUID multiplexers," Low Temperature Detectors 605, 301-304 (Feb. 2002).

[12] Battistelli, E. S. et al., "Functional description of read-out electronics for time-domain multiplexed bolometers for millimeter and sub-millimeter astronomy," J. Low Temp. Phys. 151, 908-914 (2008).

[13] Orlando, A. et al., "Antenna-coupled TES bolometer arrays for BICEP2/Keck and SPIDER," These proceedings (2010).

[14] Irwin, K. D. and Hilton, "Transition-Edge Sensors," Cryogenic Particle Detection 99, 63-149 (2005). 